\title{
An Uncommon Etiology of Small Bowel Obstruction: Gallstone Ileus
}

Akshra Verma, MD, $\mathrm{ms}^{1,2}$ Ashish Moonat, $\mathrm{MD}, \mathrm{ms}^{3}$ Adolf Lo, MD ${ }^{2,4}$

\author{
${ }^{1}$ Department of Internal Medicine, University of Illinois at Urbana-Champaign, Urbana, Illinois. \\ ${ }^{2}$ Department of Internal Medicine, Provena Medical Center, Urbana, Illinois. \\ ${ }^{3}$ Department of Surgery, Bronx Lebanon Hospital, Bronx, New York. \\ ${ }^{4}$ Department of Surgery, University of Illinois at Urbana-Champaign, Urbana, Illinois.
}

Disclosure: None of the authors have any financial relationship or conflicts of interest.

Gallstone ileus is a rare etiology of small bowel obstruction, occurring typically in the elderly population. The delay in diagnosis often results in significant morbidity and mortality. Here, we describe a case of 67 year old woman who presented with non specific abdominal symptoms and on evaluation was found to have small bowel obstruction caused by wedging of a large gallstone at the terminal ileum. Journal of Hospital Medicine 2010;5:E21-E22. @ 2010 Society of Hospital Medicine.

KEYWORDS: cholelithiasis, gallstone ileus, small bowel obstruction.

A 67-year-old female presented with nausea and nonspecific abdominal pain occurring intermittently for 2 weeks. Physical examination revealed no abdominal guarding or rigidity and was significant only for slightly hypoactive bowel sounds. Routine laboratory evaluation including liver panel was unremarkable. X-ray of the abdomen showed air fluid levels, and a diagnosis of small bowel obstruction/ileus secondary to adhesions from a previous hysterectomy was established. Conservative management with nasogastric suction, intravenous (IV) fluids and nil-by-mouth was continued for 3 days but no clinical improvement was seen. Due to continued abdominal pain, an ultrasound of the abdomen was done, which revealed cholelithiasis and chronic cholecystitis. At this point the patient was transferred to our facility and a computerized axial tomography (CAT) scan of the abdomen was done, which revealed a gallstone in the ileum (Figure 1) and the diagnosis of gallstone ileus was made. Air in the biliary tract (pneumobilia) was noted, suggesting the presence of a fistula between the gallbladder and the gastrointestinal (GI) tract. The fistula itself could not be clearly visualized. The patient was immediately taken to surgery, where small bowel exploration was done. A large gallstone completely obstructing the terminal ileum was removed by enterotomy. The gallbladder was adherent to the stomach and a cholecystogastric fistula with a gallstone coming out on the gastric end was noted. This gallstone was removed and the cholecystogastric fistula was repaired. Cholecystectomy was done at the same time. The patient recovered without any complications and has been doing well.

\section{Discussion}

Gallstone ileus accounts for approximately $1 \%$ to $2 \%$ cases of small bowel obstruction, most of which are in the elderly population. It is much more common in females as compared to males. ${ }^{1}$ Although the morbidity and mortality associated with gallstone ileus has reduced in comparison to the previous decade, probably due to the more effective usage of imaging techniques like ultrasound, CAT scan, magnetic resonance imaging (MRI), and endoscopy in the diagnostic evaluation of abdominal pain, the numbers still range between $12 \%$ to $25 \%{ }^{1,2}$ Early diagnosis plays a major role in reducing the mortality in these patients. Therefore, it is important to consider gallstone ileus in the differential diagnosis of an elderly patient presenting with bowel obstruction.

Gallstone ileus is usually associated with a biliary-enteric fistula that allows the passage of a gallstone from the gallbladder into the bowel. This gallstone gets impacted in the gastrointestinal lumen and causes mechanical bowel

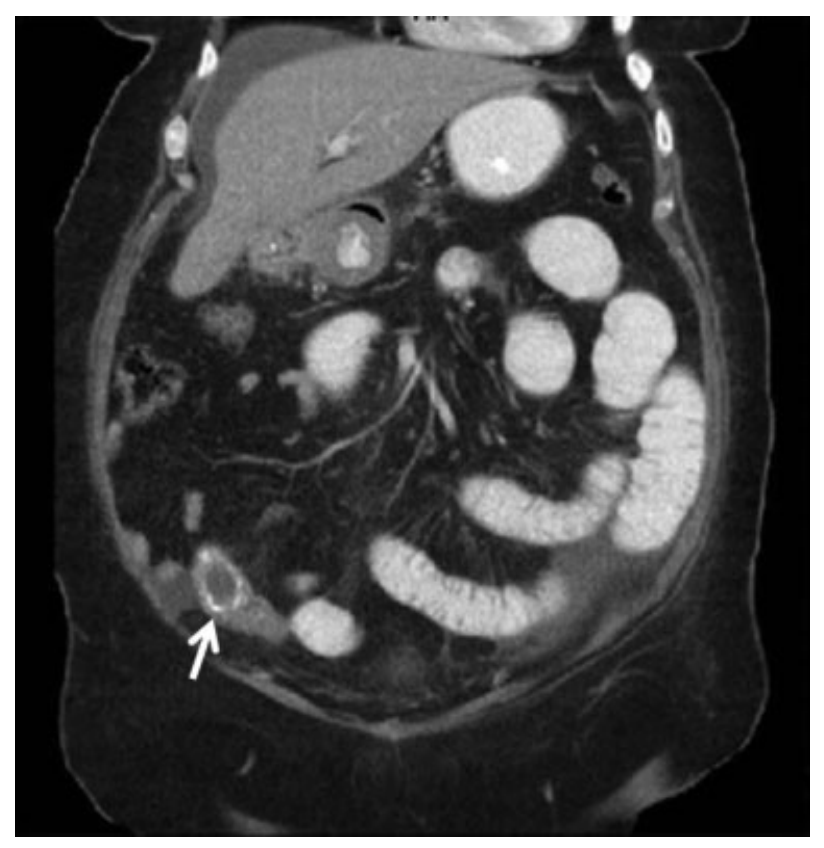

FIGURE 1. Abdomen CT showing gallstone in the terminal ileum (arrow). Abbreviation: CT, computed tomography.

2010 Society of Hospital Medicine DOI 10.1002/jhm.582 Published online in wiley InterScience (www.interscience.wiley.com). 
obstruction. The term "gallstone ileus" is a misnomer as the gallstone causes actual obstruction rather than just ileus. Considering the high prevalence of cholelithiasis, it must be realized that formation of cholecystoenteric fistula is relatively rare (about $2 \%$ in patients with cholecystitis). It is proposed that pericholecystic inflammation after an episode of cholecystitis results in the formation of adhesions between the biliary and gastrointestinal tracts. The gallstone causes pressure necrosis of the biliary wall and then erodes through it to form a fistulous communication with the adherent enteric system. Among these fistulous communications, cholecystoduodenal fistulas are the most common (60\%) while cholecystocolonic and cholecystogastric fistulas are also seen. After the biliary stone has eroded through the enteric wall, air within the intestinal tract now freely enters the biliary system, leading to an appearance of pneumobilia on imaging studies. Mirizzi syndrome, described as common hepatic duct obstruction caused by an extrinsic compression from an impacted stone in the cystic duct, is often associated with gallstone ileus. ${ }^{3}$

Once the gallstone enters the enteric tract through the fistula and traverses down the gastrointestinal tract, it causes intermittent abdominal pain, nausea, and vomiting. The symptoms may be spread over multiple days as the gallstone causes transient obstruction with its impaction and disimpaction. These obstructing gallstones that cause luminal obstruction are usually larger than $2 \mathrm{~cm}$. Majority of them will traverse the duodenum, jejunum, and small intestine, and finally get lodged in the terminal ileum (60\%), the narrowest part of the small intestine. Other sites where obstruction may occur include jejunum (16\%), duodenum, stomach, and colon. ${ }^{1}$ Bouveret's syndrome is a variant of gallstone ileus wherein the gallstone impacts in the pylorus of the stomach or the duodenum, leading to gastric outlet obstruction. $^{3}$
The diagnosis of gallstone ileus is not always straightforward and requires a high index of suspicion. It is classically described by the Rigler's triad-pneumobilia, partial or complete bowel obstruction, and ectopic gallstone, ${ }^{4}$ although often all 3 signs are not elicited. Most patients need open enterolithotomy for relief of bowel obstruction. Cholecystectomy may or may not be performed. The literature is controversial regarding the best approach of surgical management. Two surgical approaches are equally accepted: (1) a 1-stage approach, which includes enterolithotomy, cholecystectomy, and fistula repair at the same time; and (2) the other option is a 2-stage approach in which enterolithotomy is performed first and biliary surgery is performed later, if indicated. The patient's age, comorbidities, and the associated surgical risks are often used to decide between the 2 surgical approaches.

Gallstone ileus should be considered in the differential for the etiology of small bowel obstruction, especially in an elderly female known to have cholelithiasis.

\section{Address for correspondence and reprint requests:}

Akshra Verma, MD, Department of Internal Medicine, University of Illinois at Urbana-Champaign, 611 West Park St., Carle Forum Lower Level, MC-474, Urbana, IL 61801; Telephone: (217)-3833110; Fax: 217-244-0621; E-mail: averma1@illinois.edu Received 15 May 2009; revision received 5 June 2009; accepted 17 June 2009.

\section{References}

1. Reisner RM, Cohen JR. Gallstone ileus (a review of 1001 reported cases). Am Surg. 1994;60:441-446.

2. Deitz DM, Standage BA, Pinson CW, et al. Improving the outcome in gallstone ileus. Am J Surg. 1986;151:572-576.

3. Beltran MA, Csendes A, Cruces KS. The relationship of Mirizzi syndrome and cholecystoenteric fistula: validation of a modified classification. World J Surg. 2008;32(10):2237-2243.

4. Rigler LG, Borman CN, Noble JF. Gallstone obstruction: pathogenesis and roentgen manifestations. JAMA. 1941;117:1753-1759. 